\title{
AMENDMENTS
}

\section{Author Correction: 15 years of Nature Physics}

Alison Wright, Ed Gerstner, Richard Brierley, Andreas Trabesinger, May Chiao, lulia Georgescu, Bart Verberck, Luke Fleet, Abigail Klopper, Andrea Taroni, Federico Levi, Yun Li, Jan Philip Kraack, David Abergel, Stefanie Reichert, Nina Meinzer and Elizaveta Dubrovina

Correction to: Nature Physics https://doi.org/10.1038/s41567-020-01051-9, published online 1 October 2020.

In the version of this Feature originally published, in the piece 'A ghost-like quasiparticle', the first sentence of the fourth paragraph, "A dipolar quantum gas was first created in 2012, and six years later, Lauriane Chomaz and colleagues eventually reported the observation of the roton excitation", was incorrect. It should have been "While a dipolar quantum gas was first created in 2005, the fabrication of a sufficiently dipolar condensate of lanthanide atoms took longer to achieve and, eventually in 2018, Lauriane Chomaz and colleagues reported the observation of the roton excitation". The online versions of the Feature have been amended.

Published online: 24 November 2020

https://doi.org/10.1038/s41567-020-01113-y

๑) Springer Nature Limited 2020

\section{Publisher Correction: A charge-density-wave topological semimetal}

Wujun Shi (D), Benjamin J. Wieder (D), Holger L. Meyerheim, Yan Sun (D), Yang Zhang, Yiwei Li, Lei Shen (D), Yanpeng QiD, Lexian Yang, Jagannath Jena, Peter Werner, Klaus Koepernik, Stuart Parkin (iD, Yulin Chen, Claudia Felser (D),

B. Andrei Bernevig (D) and Zhijun Wang (D)

Correction to: Nature Physics https://doi.org/10.1038/s41567-020-01104-z, published online 4 January 2021.

In the version of this Article originally published online, the 'Equally contributing' statement was missing, and should have read "These authors contributed equally: Wujun Shi, Benjamin J. Wieder, Holger L. Meyerheim."; this has now been added to all versions of the Article.

Published online: 18 January 2021

https://doi.org/10.1038/s41567-021-01169-4

๑ The Author(s), under exclusive licence to Springer Nature Limited 2021 